\title{
A Cytosolic Thioredoxin Acts as a Molecular Chaperone for Peroxisome Matrix Proteins as Well as Antioxidant in Peroxisome
}

\author{
Hui $\mathrm{Du}^{1,3}$, Sunghan $\mathrm{Kim}^{2,3}$, Yoon-Sun Hur ${ }^{1}$, Myung-Sok Lee ${ }^{1}$, Suk-Ha Lee ${ }^{2}$, and Choong-III Cheon ${ }^{1, *}$
}

\begin{abstract}
Thioredoxin (TRX) is a disulfide reductase present ubiquitously in all taxa and plays an important role as a regulator of cellular redox state. Recently, a redox-independent, chaperone function has also been reported for some thioredoxins. We previously identified nodulin-35, the subunit of soybean uricase, as an interacting target of a cytosolic soybean thioredoxin, GmTRX. Here we report the further characterization of the interaction, which turns out to be independent of the disulfide reductase function and results in the co-localization of GmTRX and nodulin-35 in peroxisomes, suggesting a possible function of GmTRX in peroxisomes. In addition, the chaperone function of GmTRX was demonstrated in in vitro molecular chaperone activity assays including the thermal denaturation assay and malate dehydrogenase aggregation assay. Our results demonstrate that the target of GmTRX is not only confined to the nodulin-35, but many other peroxisomal proteins, including catalase (AtCAT), transthyretin-like protein 1 (AtTTL1), and acyl-coenzyme A oxidase 4 (AtACX4), also interact with the GmTRX. Together with an increased uricase activity of nodulin-35 and reduced ROS accumulation observed in the presence of GmTRX in our results, especially under heat shock and oxidative stress conditions, it appears that GmTRX represents a novel thioredoxin that is co-localized to the peroxisomes, possibly providing functional integrity to peroxisomal proteins.
\end{abstract}

\section{INTRODUCTION}

Thioredoxins are small, ubiquitous proteins which act as disulfide reductase, catalyzing unidirectional thiol-disulfide interchange between themselves and substrate proteins, thereby playing as a major cellular redox switch (Collet and Messens,

\footnotetext{
1Department of Biological Science, Sookmyung Women's University, Seoul 140-742, Korea, '2Department of Plant Science, Seoul National University, Seoul 151-742, Korea, ${ }^{3}$ These authors contributed equally to this work.

${ }^{*}$ Correspondence: ccheon@sookmyung.ac.kr

Received 18 September, 2014; revised 17 December, 2014; accepted 18 December, 2014; published online 15 January, 2015
}

Keywords: chaperone, peroxisome, thioredoxin, uricase
2010; Couturier et al., 2013). By far the largest family of thioredoxins is found in plants, which can be subdivided into six major groups, Trxf, Trxm, Trxx, Trxy, Trxo, and Trxh, based upon the primary amino acid sequence similarities (Gelhaye et al., 2005). The chloroplast-targeted thioredoxins, which include Trxf $-m$, $-x$, and $-y$, regulate the activity of the enzymes involved in photosynthetic carbon assimilation as well as other carbohydrate metabolisms (Lemaire et al., 2007). Trxo and a subset of Trxh are localized in the mitochondria, and they are thought to be involved in the regulation of a number of essential mitochondrial functions (Balmer et al., 2004; Gelhaye et al., 2004). Trxh, which represents the largest group of plant thioredoxins, are largely cytosolic but some non-cytosolic forms are also found in various subcellular locations including the nucleus, plasma membrane, extracellular matrices, apoplasts, and phloem saps (Meng et al., 2010; Serrato et al., 2001; Zhang et al., 2011). In line with such diverse subcellular locations, they have been implicated in a number of different cellular processes including seed germination and development, oxidative stress responses, nodule development and intercellular communication. More recently, it has been also reported that some $h$-type thioredoxins exhibit chaperone function (Lee et al., 2009; SanzBarrio et al., 2012).

We previously identified a novel $h$-type thioredoxin from soybean (Glycine max) and demonstrated its positive role in nodule development (Lee et al., 2005). The protein, tentatively named GmTRX, was confirmed to be a cytosolic thioredoxin but showed a specific interaction with nodulin-35 (N35), a nodule-specific uricase targeted to the peroxisomes (Du et al., 2010). In the present study, we set out to further characterize this interaction and its outcome. Contrary to our initial view on the role of GmTRX, our results showed that the N35 uricase was not the only target of GmTRX for the interaction, but a number of other peroxisomal enzymes were also identified as its targets. GmTRX was found co-localized to the peroxisomes with these proteins, suggesting that it may play a role in protecting these proteins from damage by oxidative stress.

\section{MATERIALS AND METHODS}

\section{Plant materials}

Arabidopsis thaliana (ecotype Columbia-0) seeds were surfacesterilized in $75 \%$ ethanol with $0.05 \%$ Tween-20 for $15 \mathrm{~min}$, washed twice with $95 \%$ ethanol and once again with $100 \%$ 
ethanol. Arabidopsis plants were grown in a growth chamber at $22^{\circ} \mathrm{C}$ under long-day conditions (16-h light/8-h dark photoperiods) and used in protoplast isolation as described previously (Yoo et al., 2007).

Bimolecular fluorescence complementation (BiFC) analysis cDNAs for the N-terminal (amino acid No. 1 to 158) and Cterminal regions (amino acid No. 158 to 238) of YFP were cloned into the p326-GFP vector after removing GFP to generate p326YFP ${ }^{\mathrm{N}}$ and $\mathrm{p} 326 \mathrm{YFP}^{\mathrm{C}}$, respectively. Full-length CDNA of the N35 was cloned into vector p326YFP ${ }^{\mathrm{N}}$ to generate N35YFP $^{N}$. Full-length cDNA or CDNA for the N-terminal (amino acid No. 1 to 40) or a C-terminal regions (amino acid No. 41 to 135) of GmTRX was cloned into p326YFP ${ }^{C}$ to generate GmTRXYFP $^{\mathrm{C}}, \mathrm{GmTRX}^{\mathrm{N}} \mathrm{NT}-\mathrm{YFP}^{\mathrm{C}}$, and GmTRX-CT-YFP ${ }^{\mathrm{C}}$, respectively. Amino acid substitution in the conserved catalytic amino acids (C59S and C62S) was carried out by PCR-mediated mutagenesis, forming GmTRX $\mathrm{dm}_{\mathrm{dm}}$ cDNAs of AtCAT3, AtTTL1, AtTTL2, $A t A P X 1$, and $A t A C X 4$ were cloned into the p326YFP ${ }^{N}$ vector. The resulting fusion constructs were then introduced into Arabidopsis protoplasts as described previously (Yoo et al., 2007).

\section{Chaperone activity}

Chaperone activity was examined using malate dehydrogenase $(\mathrm{MDH})$ or Smal. Thermal aggregation of $\mathrm{MDH}$ was performed as described (Lee et al., 2009; Park et al., 2009; Sanz-Barrio et al., 2012). $\mathrm{MDH}$ was incubated in $50 \mathrm{mM}$ HEPES-KOH $(\mathrm{pH}$ 8.0) buffer at $43^{\circ} \mathrm{C}$ with various concentrations of GmTRX (molar ratios of GmTRX to MDH of 1:1, 2:1 and 4:1). The thermal aggregation of $\mathrm{MDH}$ was determined by monitoring the increase in turbidity at $340 \mathrm{~nm}$ using a spectrophotometer. For the Smal activity assay (Santhoshkumar and Sharma, 2001), thermal denaturation was first performed at $37^{\circ} \mathrm{C}$ for $90 \mathrm{~min}$, after which DNA digestion was carried out at $25^{\circ} \mathrm{C}$ for another 90 min. GmTRX or BSA was added to 3 units of Smal in the supplied buffer.

\section{Heat treatment}

For the thermotolerance assay, the sterilized Arabidopsis seeds were plated on $0.8 \%$ agar plates of half-strength MS and grown at $22^{\circ} \mathrm{C}$ for 7 days. The plates were then sealed with parafilm, heated in a temperature-controlled water bath at $45^{\circ} \mathrm{C}$ for $2 \mathrm{~h}$, and grown under normal growth conditions for 5 days.

\section{Detection of hydrogen peroxide}

As a hydrogen peroxide $\left(\mathrm{H}_{2} \mathrm{O}_{2}\right)$ staining agent, 3,3'-diaminobenzidine (DAB) was used after dissolving in $\mathrm{H}_{2} \mathrm{O}$ and adjusting to $\mathrm{pH} 3.0$ with $\mathrm{HCl}$. The DAB solution was freshly prepared in order to avoid auto-oxidation. Two-week-old Arabidopsis seedlings grown after heat shock were immersed and infiltrated with $2 \mathrm{ml} \mathrm{DAB}$ solution under vacuum. After incubation for $4 \mathrm{~h}$ at room temperature, stained plantlets were bleached in acetic acidglycerol-ethanol $(1 / 1 / 3)(\mathrm{v} / \mathrm{v} / \mathrm{v})$ solution at $95^{\circ} \mathrm{C}$ for $15 \mathrm{~min}$, and then stored in fresh bleaching solution until photographs were taken (Guan et al., 2013). Experiments were repeated three times using more than 20 plantlets.

\section{Detection of ROS production}

Arabidopsis protoplasts were isolated and heated at $42^{\circ} \mathrm{C}$ for 10 min. The protoplasts were then incubated with $\mathrm{H}_{2}$ DCFDA at a final concentration of $5 \mu \mathrm{M}$ for $10 \mathrm{~min}$ in the dark (Gomes et al., 2005). The ROS production was visualized under a Zeiss LCSM laser confocal scanning microscope. The fluorescence intensity of dichlorofluorescein (DCF) was also measured with a fluorescence spectrometer (PerkinElmer, LS55) at room temperature with excitation at $488 \mathrm{~nm}$ and emission at $500-600 \mathrm{~nm}$.

\section{Uricase assay}

Plant extracts were isolated from 2-week-old seedlings of wildtype (Col-0), GmTRX-expressing, and $\mathrm{GmTRX}_{\mathrm{dm}}$-expressing Arabidopsis after treatment with heat shock, $\mathrm{H}_{2} \mathrm{O}_{2}$ or menadione. Each extract was added to uricase reaction mixture containing $0.1 \mathrm{mM}$ uric acid, and the uricase activity was measured as described previously (Du et al., 2010; Suzuki and Verma, 1991).

\section{RESULTS}

Interaction between GmTRX and nodulin-35 in Arabidopsis protoplasts

Despite being a cytosolic thioredoxin, GmTRX was found to interact with a peroxisomal protein, soybean uricase nodulin-35 (N35), in our previous study (Du at al., 2010). Our initial reasoning was that the actual interaction exists between a peroxisomal form of thioredoxin (TRX) and N35, and that the observed interaction merely reflects the structural similarity between peroxisomal and cytosolic TRXs. With no peroxisomal TRX having been identified either in soybean or Arabidopsis, we opted to use a mitochondrial TRX from Arabidopsis in our previous study to confirm the in vivo interaction with N35 via the bimolecular fluorescence complementation (BiFC) analysis (Du at al., 2010), because peroxisomes are most closely associated with mitochondria with active exchange of enzymes between the two organelles (Neuspiel et al., 2008). To test this possibility in root nodules, we generated transgenic nodules over-expressing either GmTRX or a peroxisomal form of TRX that was engineered by adding a peroxisomal targeting sequence 1 (PTS1; Ser-Lys-Leu) to the cytosolic GmTRX, to form GmTRX-PTS1, and compared N35 activity of the two transgenic nodules. To our surprise, the transgenic nodules expressing the cytosolic GmTRX showed uricase activity as high as that of the transgenic nodules expressing the peroxisomal GmTRX-PTS1 (data not shown).

Thus, it was speculated that even though GmTRX is a cytoplasmic protein, it might establish a functional interaction with $\mathrm{N} 35$ and both ends up in the peroxisomes. This possibility was tested by BiFC analysis using the full-length cDNAs of GmTRX and N35, and as shown in Fig. 1A, protoplasts expressing both GmTRX-YFPC and N35-YFP ${ }^{\mathrm{N}}$ exhibited fluorescence, indicating that both proteins in fact interact with each other in vivo. The BLAST sequence comparison of GmTRX with other orthologuous cytosolic TRXs revealed that it contains additional 38 amino acids at the $\mathrm{N}$-terminus (Fig. 1B). When the N-terminal extension of GmTRX (GmTRX-NT) was isolated separately and tested for binding with N35 by BiFC, it did not show any fluorescence signal, while the remainder of GmTRX (GmTRX$\mathrm{CT}$ ) containing the canonical catalytic domain of the thioredoxin family showed a strong interaction in the same assay (Fig. 1B), suggesting that the catalytic domain of GmTRX may be largely involved in the interaction. To further determine if the interaction is dependent upon the catalytic activity of GmTRX, we generated a mutant form of GmTRX ( $\left(\mathrm{GmTRX} \mathrm{X}_{\mathrm{dm}}\right)$, which was rendered catalytically inactive through substitution of both critical cysteine residues in the conserved catalytic motif (CXXC) with serines (see "Materials and Methods"), and tested its interaction with $\mathrm{N} 35$ via BiFC analysis as well. As shown in Fig. 1B, the protoplasts expressing $\mathrm{GmTRX}_{\mathrm{dm}}-\mathrm{YFP}^{\mathrm{C}}$ and N35-YFP ${ }^{\mathrm{N}}$ constructs also exhibited a strong fluorescence signal, indicating that the 
A

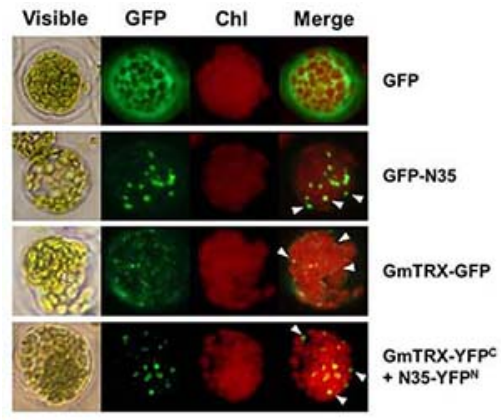

B

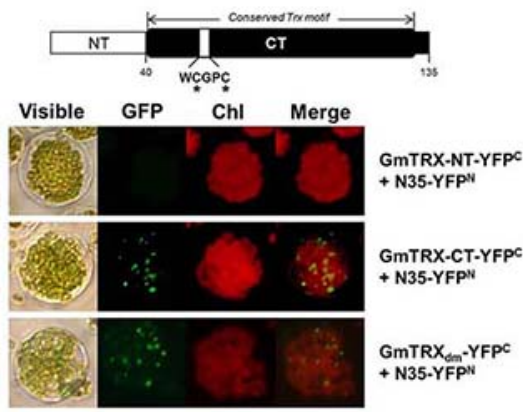

C

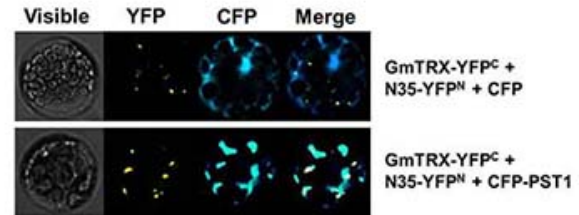

Fig. 1. Analysis of the interaction between GmTRX and nodulin-35 (N35) in Arabidopsis protoplasts. Bimolecular fluorescence complementation (BiFC) analyses of the interaction between GmTRX and N35 were performed as follows: expression of $P_{355}$-GFP as a control (GFP; A); expression of N35 fused to GFP (GFP-N35; A); expression of GmTRX fused to GFP (GmTRX-GFP; A); coexpression of $P_{355}-G_{m}$ TRX-YFPC

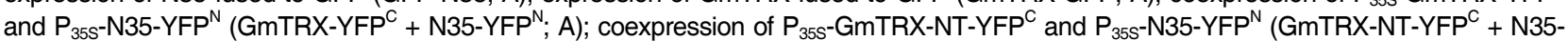

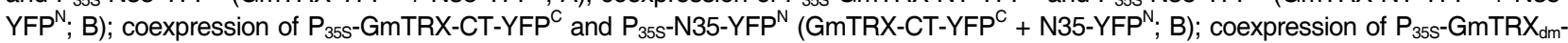

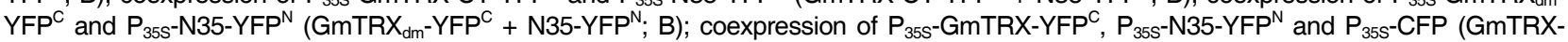

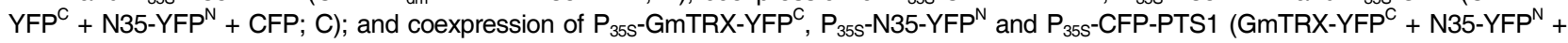
CFP-PTS1; C). White arrowheads in (A) indicate the peroxisome-like area. A schematic representation of the domain structure of GmTRX used in the BiFC analysis is shown in (B); NT, the region of amino acids from No. 1 to 40; CT, the rest of the whole protein. Conserved cysteines in the catalytic domain (C59 and C62) are indicated. GmTRX $\mathrm{dm}_{\mathrm{dm}}$ : a mutated form of GmTRX with catalytic cysteins substituted (see "Materials and Methods"). PTS1: peroxisomal targeting signal 1. Chl: autofluorescence of chloroplasts. These experiments were replicated three times with similar results.

A

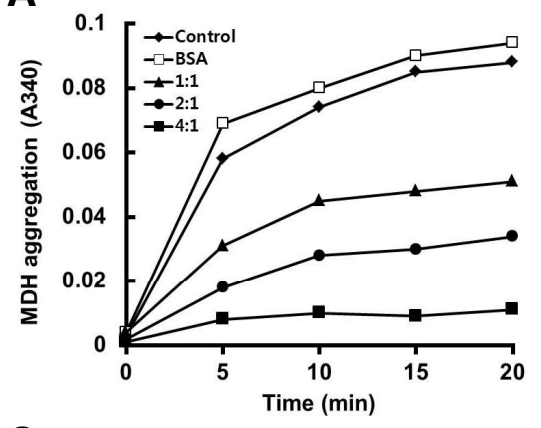

C
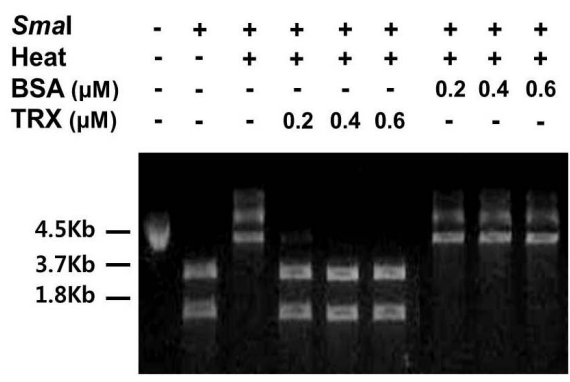

B

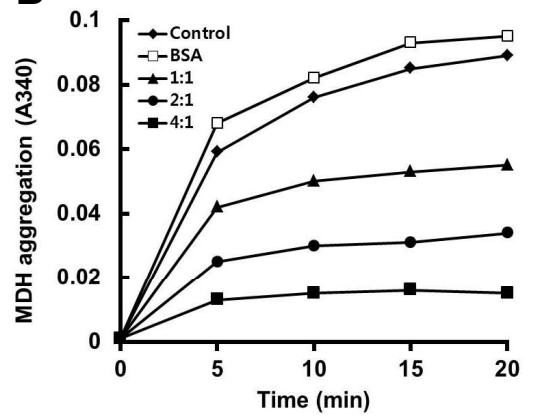

D
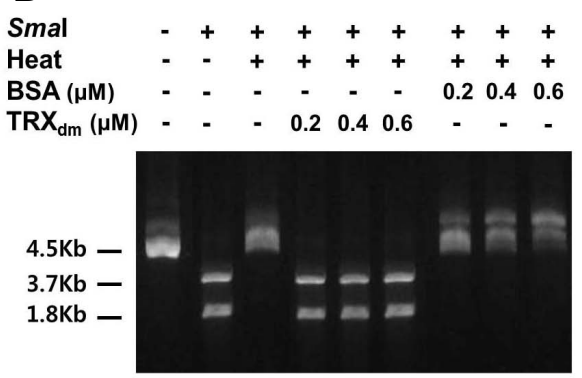

Fig. 2. Chaperone activity of GmTRX. (A, B) Holdase chaperone activity of GmTRX. Thermal aggregation of $\mathrm{MDH}$ was examined at $43^{\circ} \mathrm{C}$ in the absence (control; $\diamond$ ) or presence of $\operatorname{GmTRX}(\mathrm{A})$ or $\mathrm{GmTRX}_{\mathrm{dm}}$ (B) with the molar ratios of $\mathrm{G}$ mTRX to MDH of 1:1 ( $\mathbf{\Delta}), 2: 1$ $(\bullet)$, and 4:1 (๓). Bovine serum albumin (BSA; $\square$ ) was used as negative control. (C, D) Effects of $\operatorname{GmTRX}$ (C) or $\mathrm{GmTRX}_{\mathrm{dm}}$ (D) on the thermal denaturation of Smal as a molecular chaperone. GmTRX, GmTRX $X_{d m}$ or B $S A$ was added to a reaction mixture containing 3 units of Smal and incubated for $90 \mathrm{~min}$ at 3 $7^{\circ} \mathrm{C}$, and DNA digestion was performed at $25^{\circ} \mathrm{C}$ for another $90 \mathrm{~min}$. interaction of GmTRX with N35 occurs independent of its thiol redox activity.

The observed BiFC signal pattern for the GmTRX-N35 complex appeared to coincide with that of the GFP-N35 (Figs. 1A and $1 B$ ), suggesting that GmTRX is co-localized to the peroxisomes. To verify the location of the GmTRX-N35 complex as peroxisome, we made a construct of cyan fluorescence protein
(CFP) containing a peroxisomal targeting sequence 1 (CFPPTS1) for use as a peroxisomal marker and co-transfected Arabidopsis protoplasts with the BiFC constructs of GmTRX and N35. The result revealed that most BiFC signals from the GmTRX-N35 interaction overlapped with that of CFP-PTS1 (Fig $1 C)$. When the full-length GmTRX alone was over-expressed in the protoplasts as a GFP fusion protein (Fig. 1A), GFP fluores- 
A

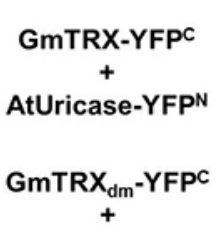

AtUricase-YFPN

GmTRX-YFPC$$
+
$$

AtCAT3-YFPN

GmTRX-YFPc

$+$

AtTTL1-YFPN

\section{GmTRX-YFPC \\ $+$ \\ AtACX4-YFPN}

GmTRX-YFPC

$+$

AtAPX1-YFPN

GmTRX-YFPc

$+$

AtTTL2-YFPN
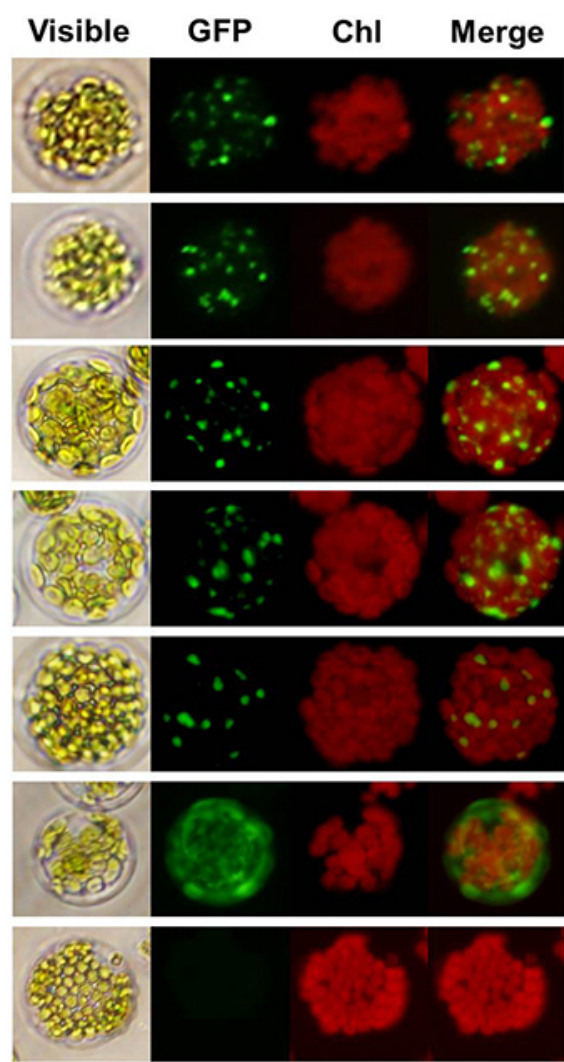

C
AtTRXh2-YFPc
$+$
N35-YFPN

AtTRXh2-YFPC

$+$

AtAPX1-YFPN

AtTRX h2-YFPC

$+$

AtCAT3-YFPN

AtTRXh2-YFPc

$+$

AtTTL1-YFPN

AtTRXh2-YFPC

$+$

AtTTL2-YFPN
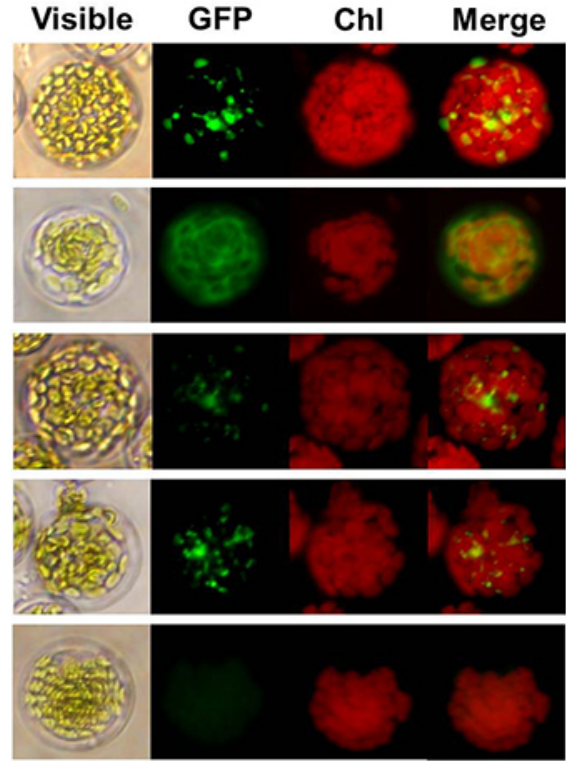

B

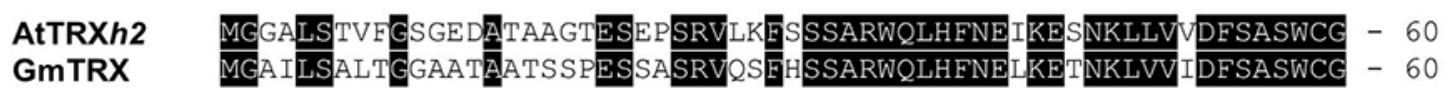

GmTRX

\section{AtTRXh2 \\ GmTRX}

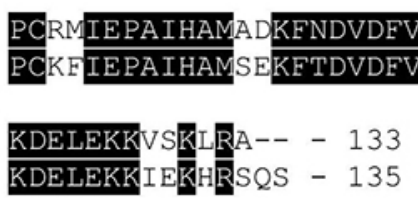

\section{AtTRXh2}

GmTRX

Fig. 3. Analysis of the interaction between GmTRX and peroxisomal proteins in Arabidopsis protoplasts. Two cytosolic proteins, AtAPX1 and AtTTL2, were used as controls. (A) BiFC analyses of the interaction between GmTRX and peroxisomal proteins were performed as follows: coexpression of $P_{355}-G m T R X-Y F P^{C}$ and $P_{355}$-AtUricase-YFP ${ }^{N}\left(G m T R X-Y F P^{C}+\right.$ AtUricase-YFP $\left.{ }^{N}\right)$; coexpression of $P_{355}-G_{m T R X} X_{d m}-Y_{F P}{ }^{C}$ and

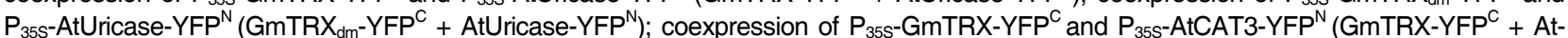

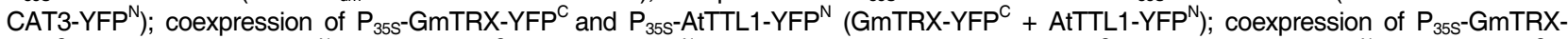

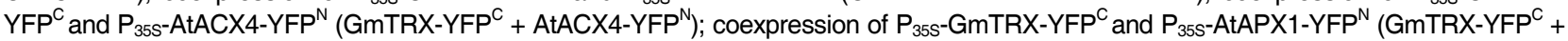

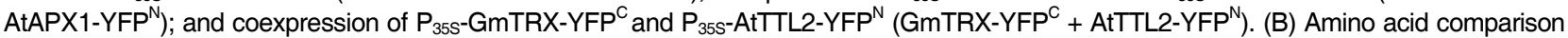
of AtTRXh2 and GmTRX. Identical amino acid residues are shaded black. Dashes indicate gaps for optimizing alignment. (C) BiFC analyses of the interaction between AtTRXh2 and peroxisomal proteins were performed as follows: coexpression of $P_{35 s}-A_{t T R X h 2-Y F P C}$ and $P_{35 s}-N_{35}$ -

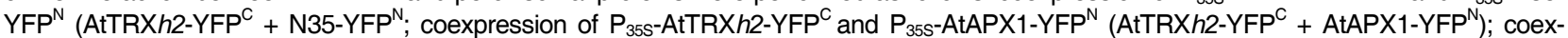

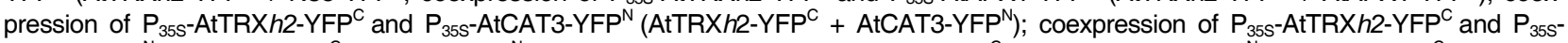

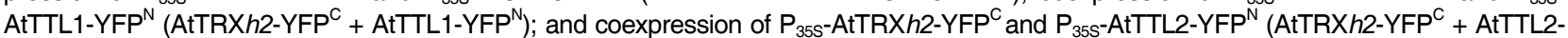
$Y_{F P}{ }^{N}$ ). Chl: autofluorescence of chloroplasts. These experiments were replicated three times with similar results.

cence was detected in these peroxisome-like area as well as in the cytoplasm. Thus, it seemed probable that GmTRX could also interact with the endogenous Arabidopsis uricase or with other peroxisomal proteins.
Property of GmTRX as a chaperone

The finding that cytosolic GmTRX interacts with N35 in a redoxindependent manner and is co-localized to peroxisomes led us to consider a possible chaperone function for GmTRX. It was previously reported that AtTDX, a thioredoxin-like protein of 
A

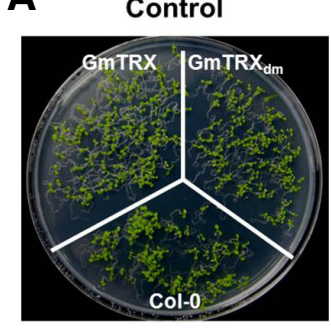

HS

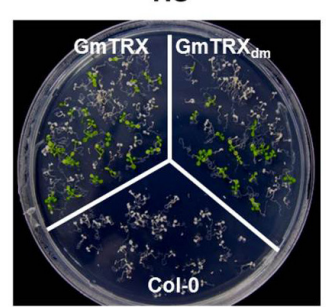

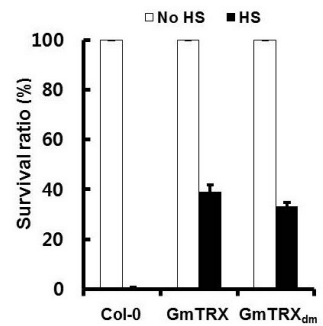

Col-0 GmTRX GmTRX
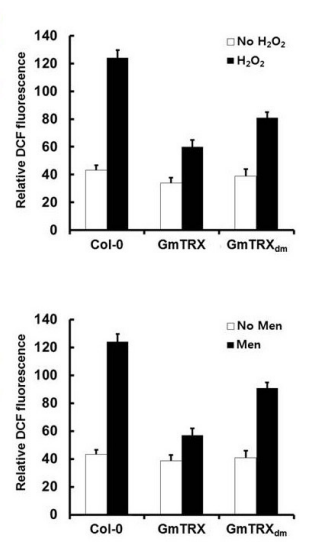

$\square$ NoHS
$-H S$

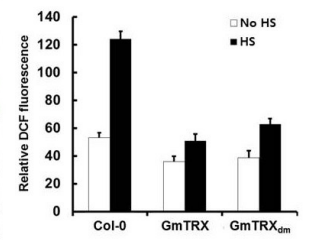

$\mathrm{H}_{2} \mathrm{O}_{2}$
Men

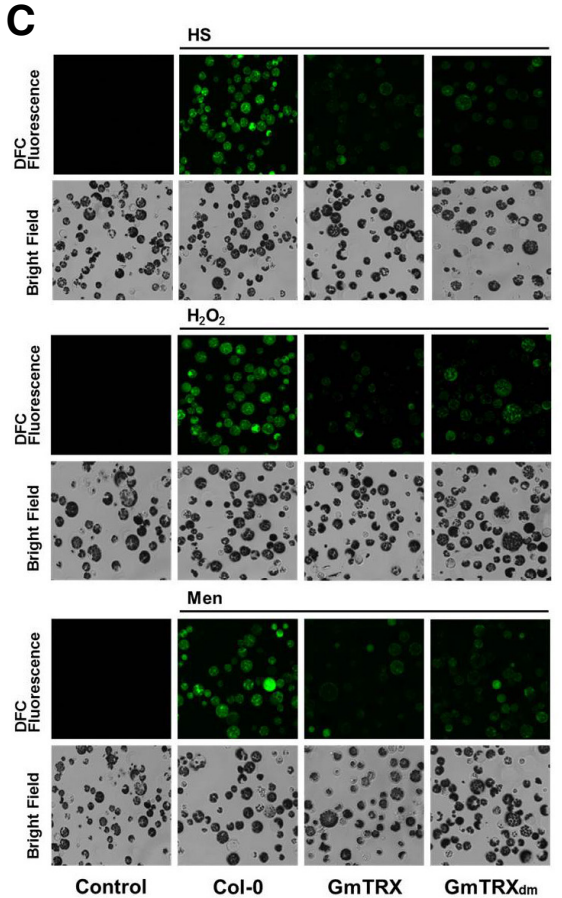

HS
B

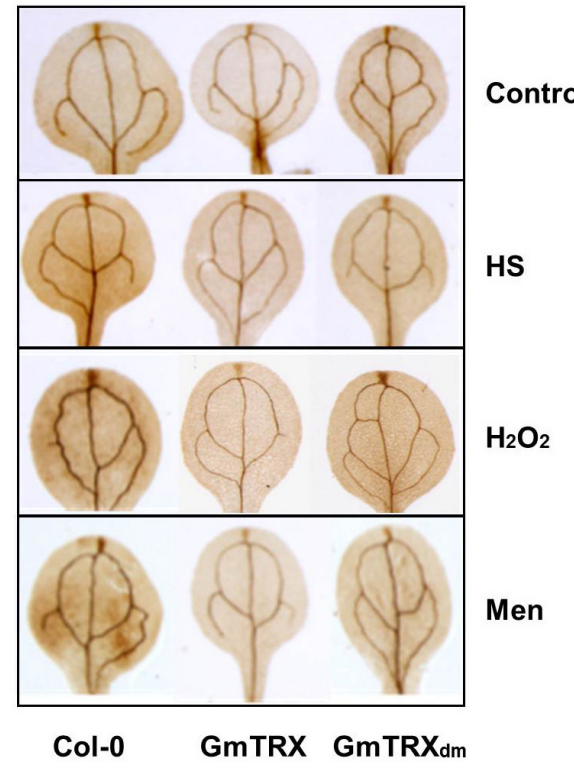

D

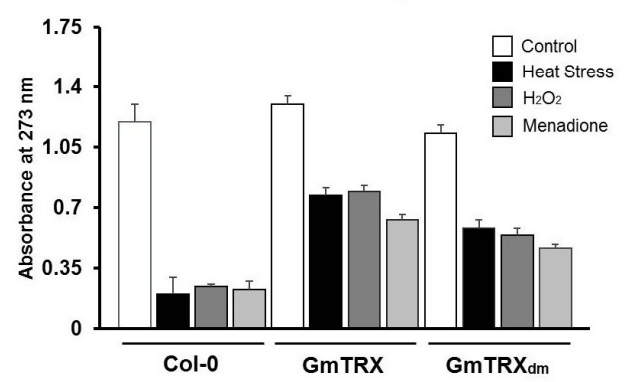

Fig. 4. Protective effects of GmTRX under oxidative stress. (A) Effects of GmTRX on Arabidopsis growth under heat shock (HS) conditions. Seven-day-old wild-type (Col-0), GmTRX-expressing, and $\mathrm{GmTRX}_{\mathrm{dm}}$-expressing Arabidopsis seedlings were subjected to heat treatment at $45^{\circ} \mathrm{C}$ for $2 \mathrm{~h}$ and allowed to recover at $22^{\circ} \mathrm{C}$ for 5 days. Survival rates of wild-type (Col-0), GmTRX-expressing, and $\mathrm{GmTRX}_{\mathrm{dm}}-\mathrm{expressing}$ Arabidopsis plants after heat shock (HS) treatment are shown. (B) The 3,3'-diaminobenzidine (DAB) staining for $\mathrm{H}_{2} \mathrm{O}_{2}$ accumulation in 2-weekold seedlings of wild-type (Col-0), GmTRX-expressing, and GmTRX dm-expressing Arabidopsis under heat shock, $\mathrm{H}_{2} \mathrm{O}_{2}$ and menadione (Men) treatment conditions. (C) ROS accumulation in Arabidopsis protoplasts. After treatment with heat shock, $\mathrm{H}_{2} \mathrm{O}_{2}$, and menadione (Men), protoplasts were kept at $22^{\circ} \mathrm{C}$ for about $1 \mathrm{~h}$ and then subjected to $5 \mu \mathrm{M} \mathrm{H}_{2}$ DCFDA for $10 \mathrm{~min}$. DCF fluorescence images were visualized by LCSM and the fluorescence intensities were measured with a fluorescence spectrometer (excitation at $488 \mathrm{~nm}$, emission at 500-600 nm). (D) Effects of GmTRX on uricase activity under oxidative stress and heat stress. Uricase activity was measured using total proteins from wild-type (Col-0), GmTRX-expressing, and $\mathrm{GmTRX}_{\mathrm{dm}}$-expressing Arabidopsis treated with $\mathrm{HS}, \mathrm{H}_{2} \mathrm{O}_{2}$, or menadione.

Arabidopsis, acts as a foldase or holdase chaperone as well as a disulfide reductase (Lee et al., 2009). Thus, the possibility that GmTRX may play a similar role as a chaperone was tested by assaying for holdase activity to reduce the thermal aggregation of malate dehydrogenase $(\mathrm{MDH})$, as shown for other typical chaperones (Lee et al., 2009; Park et al., 2009; Sanz-Barrio et al., 2012). Incubation of MDH with increasing amounts of GmTRX or $\mathrm{GmTRX}_{\mathrm{dm}}$ indeed prevented the thermal aggregation of $\mathrm{MDH}$, which showed maximum protection at a molar ratio of $4 \mathrm{GmTRX}$ (or $\mathrm{GmTRX}_{\mathrm{dm}}$ ) to $1 \mathrm{MDH}$ (Figs. $2 \mathrm{~A}$ and $2 \mathrm{~B}$ ). In addition, thermal denaturation assay on the restriction enzyme Smal (Kumar et al., 2004; Santhoshkumar and Sharma, 2001) demonstrated that GmTRX could protect the enzyme from thermal inactivation. Incubation of $S m a l$ at $37^{\circ} \mathrm{C}$ rendered complete loss of activity, while the activity was retained in the presence of GmTRX or GmTRX $X_{d m}$ (Figs. 2C and 2D). These data altogether indicate that GmTRX indeed acts as a molecular chaperone, possibly facilitating the correct folding of N35.

In order to determine if the proposed chaperone function of GmTRX is confined to nodulin-35 (N35), three additional peroxisomal proteins of Arabidopsis -- transthyretin-Like 1 (AtTTL1; Scranton et al., 2012), catalase 3 (AtCAT3; Lamberto et al., 2010) and Acyl-coenzyme A oxidase 4 (AtACX4; Hu et al., 2012) -- were chosen to test for interaction with GmTRX. All of the tested proteins were found to interact with GmTRX via BiFC analyses (Fig. 3A). The result also showed that GmTRX could 
interact with a cytosolic protein, AtAPX1 (Arent et al., 2010), while it did not interact with another cytosolic protein, AtTTL2 (Fig. 3A). These indicate that GmTRX is a cytosolic chaperone with a broad range of targets, the majority of which include peroxisomal proteins, as well as a few cytosolic proteins. Not so surprisingly, the observed role of GmTRX was also found to be conserved in Arabidopsis in that AtTRXh2, the closest orthologue of GmTRX in the Arabidopsis genome (Yamazaki et al., 2005; Fig. 3B), showed interaction with peroxisomal proteins including N35, AtCAT3, and AtTTL1 in the BiFC analysis (Fig. 3C).

\section{Enhanced protection from oxidative stresses by GmTRX} overexpression

Previously, AtTrx-h3 was shown to confer enhanced heatshock tolerance in Arabidopsis, primarily through its chaperone function (Park et al., 2009). To test if GmTRX can provide a similar biological function, seedlings of transgenic Arabidopsis plants overexpressing $\mathrm{GmTRX}$ or $\mathrm{GmTRX}_{\mathrm{dm}}$ were subjected to heat stress at $45^{\circ} \mathrm{C}$ for $2 \mathrm{~h}$. The transgenic plants showed no phenotypic differences compared with the wild type, but displayed greater heat tolerance than the wild-type plants, almost all of which did not survive over the recovery period after heat treatment (Fig. 4A).

It has been previously reported that ROS plays essential role in heat shock-induced programmed cell death in plant cells (Meyer et al., 2005; Zhang et al., 2009). Thus, we checked the accumulation of $\mathrm{H}_{2} \mathrm{O}_{2}$ in seedling leaves by 3,3'-diaminobenzidine (DAB) staining after heat shock. Under normal growth conditions, the highest level of $\mathrm{H}_{2} \mathrm{O}_{2}$ was detected in the wild-type plants, followed by the $\mathrm{GmTRX}_{\mathrm{dm}}$ transgenic plants and the GmTRX transgenic plants, in the order of the accumulation. With heat treatment, all the plants showed elevated levels of $\mathrm{H}_{2} \mathrm{O}_{2}$ but the relative level of accumulation was still far less in the transgenic plants expressing GmTRX or GmTRX dm $_{\text {(Fig. 4B). }}$

The possible role of GmTRX in the elimination of cellular ROS was also tested by applying menadione, a ROS-generating chemical, to these seedlings or treating them directly with $\mathrm{H}_{2} \mathrm{O}_{2}$. Menadione is a redox-active compound often used in the study of cellular oxidative stress as a ROS generator producing superoxide radicals $\left(\mathrm{O}_{2}^{-}\right)$and $\mathrm{H}_{2} \mathrm{O}_{2}$ (Borges et al., 2003). Again, $\mathrm{DAB}$ staining on the leaves of these plants after menadione or $\mathrm{H}_{2} \mathrm{O}_{2}$ treatment revealed that the transgenic plants expressing GmTRX or GmTRX dm had much lower levels of $\mathrm{H}_{2} \mathrm{O}_{2}$ than that of the wild-type control (Fig. 4B).

Next, the $\mathrm{H}_{2} \mathrm{O}_{2}$ accumulation was measured at the cellular level of these plants using the fluorescence probe, 2,7dichlorodihydrofluorescein (DCFH), which is converted to the highly fluorescent dichlorofluorescein (DCF) in the presence of $\mathrm{H}_{2} \mathrm{O}_{2}$ (Bartsch et al., 2008; Vacca et al., 2004). Protoplasts were isolated from these plants and measured for DCF fluorescence after treatment with heat, menadione or $\mathrm{H}_{2} \mathrm{O}_{2}$. Similar to the DAB staining results of the seedling leaves, the protoplasts derived from $\mathrm{GmTRX}$ - or $\mathrm{GmTRX}_{\mathrm{dm}}$ - transgenic plants showed less accumulation of $\mathrm{H}_{2} \mathrm{O}_{2}$ than those isolated from wild-type Arabidopsis (Fig. 4C).

Increased uricase activity resulting from the interaction with GmTRX in vitro was already observed in our previous study (Du et al., 2010). As our present study suggests that this enhancement was likely due to a redox-independent, chaperone function of GmTRX, we examined the effects of GmTRX and $\mathrm{GmTRX}_{\mathrm{dm}}$ overexpression on the endogenous uricase activity under oxidative stress conditions. The amino acids sequence of Arabidopsis uricase shows $68 \%$ identity with soybean N35. As expected, Arabidopsis uricase was able to interact with both
GmTRX and GmTRX dm $_{\text {in }}$ Arabidopsis protoplasts (Fig. 3A). Under normal growth conditions, wild-type Arabidopsis plants and the GmTRX transgenic lines showed similar uricase activity However, after heat shock, a sharp reduction in the uricase activity was observed in wild-type plants, whereas the GmTRXand $\mathrm{GmTRX}_{\mathrm{dm}}$ - expressing plants showed that the uricase activity still retained up to $60 \%$ and $50 \%$ of the original level detected in unstressed plants, respectively (Fig. 4D). Similarly, treatment with $\mathrm{H}_{2} \mathrm{O}_{2}$ or menadione resulted in about $70-80 \%$ reduction of the enzyme activity in the wild-type plants, whereas the reduction of the enzyme activity in GmTRX-transgenic plants under the same conditions was only about $30-40 \%$ (Fig. 4D). Therefore, all these data consistently demonstrate a positive role played by GmTRX in protecting cellular components from oxidative stress, and the protection mechanism appears to be independent of its oxidoreductase activity.

\section{DISCUSSION}

As a thiol-disulfide oxidoreductase participating in the cellular redox signaling pathways, thioredoxins interact with many different target proteins to carry out their dynamic regulation of structure and function. Over the last few years, a number of new targets of thioredoxins have been identified in plants (Arent et al., 2010; Courteille et al., 2013).

We also identified the nodulin-35 (N35) to be an interacting partner of GmTRX (Du et al., 2010). Our initial interpretation of the observed interaction between GmTRX and the peroxisometargeted N35 was that it was simply the reflection of a bona fide interaction between N35 and a yet-to-be identified peroxisomal form of thioredoxin. Many thioredoxins have been found in different subcellular compartments of plant cells, including chloroplasts, mitochondria, and the cytoplasm (Kern et al., 2003; Nuruzzaman et al., 2012; Zhang et al., 2011), but no thioredoxins have been found to be located in peroxisomes until now. While our efforts to identify possible candidates for the peroxisomal thioredoxin in the soybean or Arabidopsis genome have been futile, our results showed that the cytoplasmic GmTRX was localized to the peroxisomes, together with N35 (Fig. 1). This interaction did not appear to be dependent upon the conventional catalytic activity of GmTRX since the catalytically inactive mutant form of GmTRX, GmTRX $x_{d m}$, also exhibited interaction with nodulin-35 and co-localization to the peroxisomes. It is possible that GmTRX may interact with many peroxisomal proteins in the cytoplasm, followed by their targeting to peroxisomes and that the peroxisomal protein with peroxisome targeting signal aids in translocation of the GmTRXprotein complex into the peroxisome. Another possibility is that GmTRX is somehow transported to the peroxisome wherein it then binds to the peroxisomal proteins.

Within peroxisomes, GmTRX could also provide the additional function of protecting the proteins from oxidative damages, as indicated by the results with GmTRX-transgenic plants (Fig. 4). An interesting aspect of the observed reduction of ROS by GmTRX is that it did not seem to require the conventional oxidoreductase activity of TRX, thus the catalytically inactive mutant GmTRX $X_{d m}$ was as effective as the wild-type GmTRX in providing protection against ROS and heat stress, as well as in preserving the activity of peroxisomal enzymes such as uricase under such conditions. Hence, the observed protection by GmTRX against oxidative stress may be achieved indirectly through its chaperone function, stabilizing and thus enhancing the activity of ROS scavenging enzymes, with which GmTRX associates. Identification of catalase 3 (AtCAT3) as one of the 
interacting proteins with GmTRX seems to support this scenario. Recent reports of the redox-independent, chaperone function of thioredoxins in E. coli, tobacco (N. tabacum), and Arabidopsis (Kern et al., 2003 ; Kthiri et al., 2008; Lee et al., 2009; Park et al., 2009; Sanz-Barrio et al., 2012) have provided a new insight into their diverse physiological roles. A novelty in our present finding is the potential role of GmTRX as a cytoplasmic chaperone largely specific for proteins targeted to peroxisomes. In addition to nodulin-35, we showed that other peroxisomal proteins were also the interacting targets of GmTRX. Moreover, AtTRXh2, the closest ortholog of GmTRX in the Arabidopsis genome, according to amino acid sequence homology comparison, was also found to interact with nodulin-35 and co-localized to the peroxisomes in our BiFC analysis (Fig. 3B). Therefore, the role of $\mathrm{Trx}$ as a specific interacting component for peroxisomal proteins may be a widely conserved mechanism at least in plants.

In summary, our present study demonstrated a prospect of a multi-functional thioredoxin, possibly acting both as a chaperone and a disulfide reductase and plays a key role in preserving the functional integrity of key enzymes in peroxisomes, especially under oxidative stress conditions.

\section{ACKNOWLEDGMENTS}

This research was supported by the SRC Research Center for Women's Diseases of Sookmyung Women's University (2011).

\section{REFERENCES}

Arent, S., Christensen, C.E., Pye, V.E., Nørgaard, A., and Henriksen, A. (2010). The multifunctional protein in peroxisomal betaoxidation: structure and substrate specificity of the Arabidopsis thaliana protein MFP2. J. Biol. Chem. 285, 24066-24077.

Balmer, Y., Vensel, W.H., Tanaka, C.K., Hurkman, W.J., Gelhaye, E., Rouhier, N., Jacquot, J.P., Manieri, W., Schürmann, P., Droux, M., et al. (2004). Thioredoxin links redox to the regulation of fundamental processes of plant mitochondria. Proc. Natl. Acad. Sci. USA 101, 2642-2647.

Bartsch, S., Monnet, J., Selbach, K., Quigley, F., Gray, J., von Wettstein, D., Reinbothe, S., and Reinbothe, C. (2008). Three thioredoxin targets in the inner envelope membrane of chloroplasts function in protein import and chlorophyll metabolism. Proc. Natl. Acad. Sci. USA 105, 4933-4938.

Borges, A.A., Borges-Perez, A., and Fernandez-Falcon M. (2003). Effect of menadione sodium bisulfite, an inducer of plant defenses, on the dynamic of banana phytoalexin accumulation during pathogenesis. J. Agric. Food Chem. 27, 5326-5328.

Collet, J.F., and Messens, J. (2010). Structure, function, and mechanism of thioredoxin proteins. Antioxid. Redox Signal. 13, 1205-1216

Courteille, A., Vesa, S., Sanz-Barrio, R., Cazalé, A.C., BecuweLinka, N., Farran, I., Havaux, M., Rey, P., and Rumeau, D. (2013). Thioredoxin $\mathrm{m} 4$ controls photosynthetic alternative electron pathways in Arabidopsis. Plant Physiol. 161, 508-520.

Couturier, J., Chibani, K., Jacquot, J.P., and Rouhier, N. (2013). Cysteine-based redox regulation and signaling in plants. Front. Plant Sci. 4, 105.

Du, H., Kim, S., Nam, K.H., Lee, M.S., Son, O., Lee, S.H., and Cheon, C.I. (2010). Identification of uricase as a potential target of plant thioredoxin: Implication in the regulation of nodule development. Biochem. Biophys. Res. Commun. 397, 22-26.

Gelhaye, E., Rouhier, N., Gérard, J., Jolivet, Y., Gualberto, J., Navrot, N., Ohlsson, P.I., Wingsle, G., Hirasawa, M., Knaff, D.B., et al. (2004). A specific form of thioredoxin $h$ occurs in plant mitochondria and regulates the alternative oxidase. Proc. Natl. Acad. Sci. USA 101, 14545-14550.

Gelhaye, E., Rouhier, N., Navrot, N., and Jacquot, J.P. (2005). The plant thioredoxin system. Cell Mol. Life Sci. 62, 24-35.

Gomes, A., Fernandes, E., and Lima, J.L. (2005). Fluorescence probes used for detection of reactive oxygen species. J. Biochem. Biophys. Methods 65, 45-80.
Guan, Q., Lu, X., Zeng, H., Zhang, Y., and Zhu, J. (2013). Heat stress induction of miR398 triggers a regulatory loop that is critical for thermotolerance in Arabidopsis. Plant J. 74, 840-851.

$\mathrm{Hu}$, J., Baker, A., Bartel, B., Linka, N., Mullen, R.T., Reumann, S., and Zolman, B.K. (2012). Plant peroxisomes: biogenesis and function. Plant Cell 24, 2279-2303.

Kern, R., Malki, A., Holmgren, A., and Richarme, G. (2003). Chaperone properties of Escherichia coli thioredoxin and thioredoxin reductase. Biochem. J. 371, 965-972.

Kthiri, F., Le, H.T., Tagourti, J., Kern, R., Malki, A., Caldas, T., Abdallah, J., Landoulsi, A., and Richarme, G. (2008). The thioredoxin homolog YbbN functions as a chaperone rather than as an oxidoreductase. Biochem. Biophys. Res. Commun. 374, 668-672.

Kumar, J.K., Tabor, S., and Richardson, C.C. (2004). Proteomic analysis of thioredoxin-targeted proteins in Escherichia coli. Proc. Natl. Acad. Sci. USA. 101, 3759-3764.

Lamberto, I., Percudani, R., Gatti, R., Folli, C., and Petrucco, S. (2010). Conserved alternative splicing of Arabidopsis transthyretin-like determines protein localization and $S$-allantoin synthesis in peroxisomes. Plant Cell 22, 1564-1574.

Lee, M.Y., Shin, K.H., Kim, Y.K., Suh, J.Y., Gu, Y.Y., Kim, M.R., Hur, Y.S., Son, O., Kim, J.S., Song, E., et al. (2005). Induction of thioredoxin is required for nodule development to reduce reactive oxygen species levels in soybean roots. Plant Physiol. 139, $1881-1889$

Lee, J.R., Lee, S.S., Jang, H.H., Lee, Y.M., Park, J.H., Park, S.C., Moon, J.C., Park, S.K., Kim, S.Y., Lee, S.Y., et al. (2009). Heatshock dependent oligomeric status alters the function of a plantspecific thioredoxin-like protein, AtTDX. Proc. Natl. Acad. Sci. USA 106, 5978-5983.

Lemaire, S.D., Michelet, L., Zaffagnini, M., Massot, V., and Issakidis-Bourguet, E. (2007). Thioredoxins in chloroplasts. Curr. Genet. 51, 343-365

Meng, L., Wong, J.H., Feldman, L.J., Lemaux P.G., and Buchanan, B.B. (2010). A membrane-associated thioredoxin required for plant growth moves from cell to cell, suggestive of a role in intercellular communication. Proc. Natl. Acad. Sci. USA 107, 39003905.

Meyer, Y., Reichheld, J.P., and Vignols, F. (2005). Thioredoxins in Arabidopsis and other plants. Photosynth. Res. 86, 419-433.

Neuspiel, M., Schauss, A.C., Braschi, E., Zunino, R., Rippstein, P. Rachubinski, R.A., Andrade-Navarro, M.A., and McBride, H.M (2008). Cargo-selected transport from the mitochondria to peroxisomes is mediated by vesicular carriers. Curr. Biol. 18, 102108.

Nuruzzaman, M., Sharoni, A.M., Satoh, K., Al-Shammari, T., Shimizu, T., Sasaya, T., Omura, T., and Kikuchi, S. (2012). The thioredoxin gene family in rice: genome-wide identification and expression profiling under different biotic and abiotic treatments. Biochem. Biophys. Res. Commun. 423, 417-423.

Park, S.K., Jung, Y.J., Lee, J.R., Lee, Y.M., Jang, H.H., Lee, S.S., Park J.H., Kim, S.Y., Moon, J.C., Lee, S.Y., et al. (2009). Heat-shock and redox-dependent functional switching of an h-type Arabidopsis thioredoxin from a disulfide reductase to a molecular chaperone. Plant Physiol. 150, 552-561.

Santhoshkumar, P., and Sharma, K.K. (2001). Analysis of alphacrystallin chaperone function using restriction enzymes and citrate synthase. Mol. Vis. 7, 172-177.

Sanz-Barrio, R., Fernández-San Millán, A., Carballeda, J., CorralMartínez, P., Seguí-Simarro, J.M., and Farran, I. (2012). Chaperone-like properties of tobacco plastid thioredoxins $f$ and $m$. J. Exp. Bot. 63, 365-379.

Scranton, M.A., Yee, A., Park, S.Y., and Walling, L.L. (2012). Plant leucine aminopeptidases moonlight as molecular chaperones to alleviate stress-induced damage. J. Biol. Chem. 287, 1840818417.

Serrato, A.J., Crespo, J.L., Florencio, F.J., and Cejudo, F.J. (2001). Characterization of two thioredoxins $h$ with predominant localization in the nucleus of aleurone and scutellum cells of germinating wheat seeds. Plant Mol. Biol. 46, 361-371.

Suzuki, H., and Verma, D.P. (1991). Soybean Nodule-Specific Uricase (Nodulin-35) Is Expressed and Assembled into a Functional Tetrameric Holoenzyme in Escherichia coli. Plant Physiol. 95, 384-389.

Vacca, R.A., de Pinto, M.C., Valenti, D., Passarella, S., Marra, E, and De Gara, L. (2004). Production of reactive oxygen species, 
alteration of cytosolic ascorbate peroxidase, and impairment of mitochondrial metabolism are early events in heat shockinduced programmed cell death in tobacco Bright-Yellow 2 cells. Plant Physiol. 134, 1100-1112.

Yamazaki, D., Motohashi, K., Kasama, T., Hara, Y., and Hisabori, T. (2005). Target proteins of the cytosolic thioredoxins in Arabidopsis thaliana. Plant Cell Physiol. 45, 18-27.

Yoo, S.D., Cho, Y.H., and Sheen, J. (2007). Arabidopsis mesophyll protoplasts: a versatile cell system for transient gene expression analysis. Nat. Protoc. 2, 1565-1572.
Zhang, L., Li, Y., Xing, D., and Gao, C. (2009). Characterization of mitochondrial dynamics and subcellular localization of ROS reveal that $H s f A 2$ alleviates oxidative damage caused by heat stress in Arabidopsis. J. Exp. Bot. 60, 2073-2091.

Zhang, C.J., Zhao, B.C., Ge, W.N., Zhang, Y.F., Song, Y., Sun, D.Y., and Guo, Y. (2011). An apoplastic h-type thioredoxin is involved in the stress response through regulation of the apoplastic reactive oxygen species in rice. Plant Physiol. 157, 18841899. 